\title{
Schwinger model simulations with dynamical overlap fermions
}

\author{
Wolfgang Bietenholz* \\ NIC/DESY Zeuthen, Platanenallee 6 \\ D-15738 Zeuthen, Germany \\ E-mail: bietenhodifh. de
}

\section{Stanislav Shcheredin}

Fakultät für Physik, Univerisität Bielefeld

D-33615 Bielefeld, Germany

E-mail: shchered@physik.hu-berlin.de

\section{Jan Volkholz}

Institut für Physik, Humboldt-Universität zu Berlin

Newtonstr. 15, D-12489 Berlin, Germany

E-mail: volkholz@physik.hu-berlin.de

\begin{abstract}
We present simulation results for the 2-flavour Schwinger model with dynamical overlap fermions. In particular we apply the overlap hypercube operator at seven light fermion masses. In each case we collect sizable statistics in the topological sectors 0 and 1 . Since the chiral condensate $\Sigma$ vanishes in the chiral limit, we observe densities for the microscopic Dirac spectrum, which have not been addressed yet by Random Matrix Theory (RMT). Nevertheless, by confronting the averages of the lowest eigenvalues in different topological sectors with chiral RMT in unitary ensemble we obtain - for the very light fermion masses — values for $\Sigma$ that follow closely the analytical predictions in the continuum.
\end{abstract}

The XXV International Symposium on Lattice Field Theory

July 30 - August 42007

Regensburg, Germany

${ }^{*}$ Speaker. 


\section{The Schwinger model}

The Schwinger model [1] describes Dirac fermions in $d=2$, interacting through a $U(1)$ gauge field. In the Euclidean plane the Lagrangian reads

$$
\mathscr{L}\left(\bar{\psi}, \psi, A_{\mu}\right)=\bar{\psi}(x)\left[\gamma_{\mu}\left(i \partial_{\mu}+g A_{\mu}\right)+m\right] \psi(x)+\frac{1}{2} F_{\mu \nu}(x) F_{\mu v}(x) .
$$

This is a popular toy model for QCD - for instance it is endowed with confinement. As a qualitative difference, however, there is no spontaneous chiral symmetry breaking. For $N_{f}$ degenerate flavours of mass $m$ the chiral condensate is given by ${ }^{1}$

$$
\Sigma(m) \equiv-\langle\bar{\psi} \psi\rangle \propto\left(\frac{m^{N_{f}-1}}{\beta}\right)^{1 /\left(N_{f}+1\right)} \quad\left(\beta=1 / g^{2}\right) .
$$

Here we consider $N_{f}=2$. In this case, there are analytical evaluations (using low energy assumptions) for the above proportionality constant in the case of light fermions $(m \ll 1 / \sqrt{\beta})$,

$$
\Sigma(m)= \begin{cases}0.372(m / \beta)^{1 / 3} & {[2]} \\ 0.388(m / \beta)^{1 / 3} & {[3]}\end{cases}
$$

\section{Lattice formulation}

We investigate the lattice formulation with compact link variables $U_{\mu, x} \in U(1)$, and with the plaquette gauge action. For the fermions we employ an overlap hypercube fermion (overlap-HF) Dirac operator of the form (in lattice units)

$$
D_{\mathrm{ovHF}}(m)=\left(1-\frac{m}{2}\right) D_{\mathrm{ovHF}}^{(0)}+m, \quad D_{\mathrm{ovHF}}^{(0)}=1+\left(D_{\mathrm{HF}}-1\right) / \sqrt{\left(D_{\mathrm{HF}}^{\dagger}-1\right)\left(D_{\mathrm{HF}}-1\right)} .
$$

$D_{\text {ovHF }}^{(0)}$ obeys the (simplest) Ginsparg-Wilson relation. Unlike the standard overlap operator with a Wilson kernel [4], we insert the truncated perfect hypercube fermion operator $D_{\mathrm{HF}}$ [5]. It involves couplings to nearest neighbour sites, and over plaquette diagonals (in the latter case gauging averages over the shortest lattice paths). By construction this kernel is approximately chiral already, and the overlap formula amounts to a correction that renders chirality exact. ${ }^{2}$

The overlap-HF has been applied in quenched QCD [7], and the HF was also used dynamically in finite temperature QCD [8]. In the 2-flavour Schwinger model $D_{\text {ovHF }}$ has been first simulated with quenched re-weighted configurations $[6,9]$. Compared to the standard overlap operator there is some computational overhead in the kernel, but $D_{\text {ovHF }}$ has the following virtues [6]:

- Faster convergence in the polynomial evaluation of $D_{\text {ovHF}}$. Moreover the limitation to the use of low polynomials also improves the numerical stability.

- Higher degree of locality and approximate rotation symmetry.

- Improved scaling behaviour.

All these virtues are based on the similarity of the kernel to the overlap operator [5],

$$
D_{\mathrm{ovHF}} \approx D_{\mathrm{HF}} .
$$

\footnotetext{
${ }^{1}$ For $N_{f}=1$ the non-vanishing value $\Sigma(0)=\left(e^{\gamma} / 2 \pi^{3 / 2}\right) g \simeq 0.16 g$ originates from an axial anomaly and therefore from explicit chiral symmetry breaking (hence there is no contradiction to the Mermin-Wagner theorem).

${ }^{2}$ To be precise, we use the chirally-optimised hypercube fermion (CO-HF) of Ref. [6]. This is optimal for our algorithm to be described in Section 3.
} 


\section{The simulation}

Here we report on HMC simulations, which are also facilitated by the property (2.2). Our algorithmic concept follows the simplified HMC force for improved staggered fermions of the HF-type [10]. The fermionic force of the standard HMC algorithm

$$
\bar{\psi} Q_{\mathrm{ovHF}}^{-1}\left[Q_{\mathrm{ovHF}}^{-1} \frac{\partial Q_{\mathrm{ovHF}}}{\partial A_{x, \mu}}+\frac{\partial Q_{\mathrm{ovHF}}}{\partial A_{x, \mu}} Q_{\mathrm{ovHF}}^{-1}\right] Q_{\mathrm{ovHF}}^{-1} \psi,
$$

with the Hermitian operator $Q_{\mathrm{ovHF}}=\gamma_{5} D_{\mathrm{ovHF}}$, is simplified to

$$
\bar{\psi} Q_{\mathrm{ovHF}, \varepsilon}^{-1}\left[Q_{\mathrm{ovHF}, \varepsilon}^{-1} \frac{\partial Q_{\mathrm{HF}}}{\partial A_{x, \mu}}+\frac{\partial Q_{\mathrm{HF}}}{\partial A_{x, \mu}} Q_{\mathrm{ovHF}, \varepsilon}^{-1}\right] Q_{\mathrm{ovHF}, \varepsilon}^{-1} \psi .
$$

$Q_{\mathrm{ovHF}, \varepsilon}$ approximates $Q_{\mathrm{ovHF}}$ to a moderate (absolute) precision of $\varepsilon=10^{-5}$. This approximation is useful and cheap thanks to relation (2.2) (which does not apply for the standard overlap operator). The Metropolis accept/reject step uses $Q_{\mathrm{ovHF}}$ to machine precision $\left(10^{-16}\right)$, which renders the algorithm exact. Our first experience at $\beta=5$ on a $16 \times 16$ lattice, with trajectory length $\tau=$ $1 / 8=20 \cdot \Delta \tau$, was reported in Ref. [11]. Applying the Sexton-Weingarten integration scheme [12], we have meanwhile a compelling confirmation of acceptance rates in the range $0.3 \ldots 0.5$ for the masses $m=0.01 \ldots 0.24$. Acceptance rates for the special case $Q_{\mathrm{ovHF}, \varepsilon} \equiv Q_{\mathrm{HF}}$ were also given in Ref. [13]. Our results show a remarkable stability in $m$ down to very light fermions. This holds for the total computing effort as well; note that the magnitude of the leading non-zero Dirac eigenvalue stabilises due to the finite size. In Ref. [11] we demonstrated that reversibility holds to a good precision. The degree of locality is stable in $m$ and strongly improved, even compared to the free standard overlap fermion, where the couplings decay as $\exp (-r)(r$ being the taxi driver distance between source and sink). For the free overlap-HF this decay is accelerated to $\exp (-1.5 r)$. At $\beta=5$ it slows down only slightly to $\exp (-1.45 r)$, with hardly any dependence on the masses that we investigated [11].

\section{Results}

In view of the $\varepsilon$-regime, we simulated at 7 fermion masses and collected data in the sectors with topological charge $v=0$ and $|v|=1$ (index of $D_{\mathrm{ovHF}}$ ). The corresponding statistics and the mean values of the leading non-zero eigenvalue $\lambda_{1}$ of the Dirac operator - stereographically projected onto a line - are given in Table 1 .

Chiral RMT has been worked out for the case of a non-vanishing chiral condensate $\Sigma$ in the chiral limit. This yields predictions for the low lying Dirac eigenvalues [16] in the $\varepsilon$-regime, which apply well in QCD $[14,15]$. We show in Figure 1 the measured cumulative densities in our case, in the topologically trivial sector. This is compared to the RMT predictions for the parameters we are using (we refer to the unitary ensemble; the corresponding formulae are summarised in the second work quoted in Ref. [15]). They converge in terms of the dimensionless rescaled eigenvalue $\zeta_{1}=\lambda_{1} \Sigma V$ for very light or very heavy masses, where the latter limit corresponds to the quenched case. In the chiral limit this is obviously inapplicable in our situation. The plot in Figure 1 on the right also shows that the shape of the density for $\zeta_{1}$ at finite mass does not match the RMT predictions. Instead we see a stabilisation in the eigenvalue $\lambda_{1}$ itself (in a fixed volume $V$ ). 


\begin{tabular}{|c||r|c||r|c||c||c|}
\hline$m$ & $v=0$ & $\left\langle\lambda_{1, v=0}\right\rangle$ & $|v|=1$ & $\left\langle\lambda_{1, v \mid=1}\right\rangle$ & $\begin{array}{c}\text { total } \\
\text { statistics }\end{array}$ & $\begin{array}{c}\text { topological } \\
\text { transitions }\end{array}$ \\
\hline \hline 0.01 & 2079 & $0.1328(7)$ & 584 & $0.1735(10)$ & 2663 & 3 \\
\hline 0.03 & 1131 & $0.1311(18)$ & 563 & $0.1737(24)$ & 1668 & 2 \\
\hline 0.06 & 752 & $0.1254(24)$ & 711 & $0.1728(20)$ & 1398 & 5 \\
\hline 0.09 & 957 & $0.1157(22)$ & 546 & $0.1713(24)$ & 1504 & 7 \\
\hline 0.12 & 699 & $0.1082(28)$ & 532 & $0.1664(26)$ & 1505 & 8 \\
\hline 0.18 & 830 & $0.1076(28)$ & 609 & $0.1660(24)$ & 1493 & 13 \\
\hline 0.24 & 639 & $0.1096(28)$ & 1030 & $0.1642(18)$ & 1757 & 17 \\
\hline
\end{tabular}

Table 1: Our statistics for seven fermion masses in the sectors with topological charge $v=0$ and $|v|=1$. The (stereographically projected) leading non-zero eigenvalue $\lambda_{1}$ of the Dirac operator is measured separately in each sector.
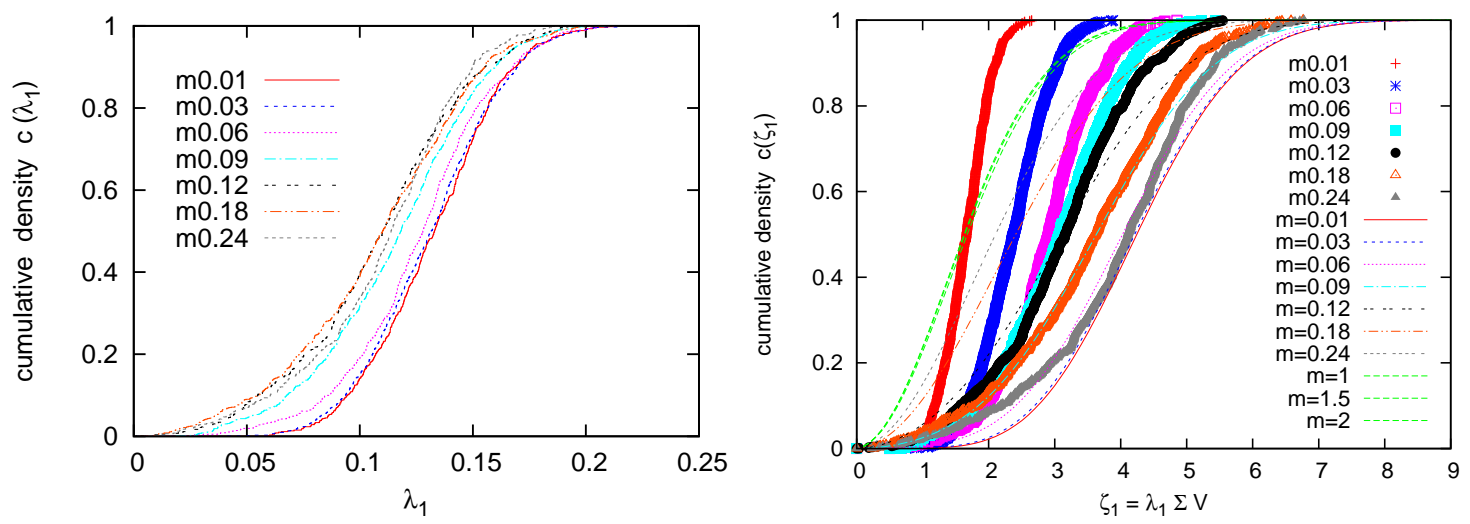

Figure 1: On the left: the cumulative densities of the lowest Dirac eigenvalues for different fermion masses. On the right: the chiral RMT prediction with the corresponding parameters, which differs from the data as expected. The RMT curves converge for $\zeta_{1} \equiv \lambda_{1} \Sigma V$ in the limits $\mu \equiv m \Sigma V \rightarrow 0$ and $\mu \rightarrow \infty$, whereas in the measured data the density of $\lambda_{1}$ stabilises in the chiral limit.

In this setting a total density $\rho(\lambda) \propto \lambda^{1 / 3}$ is consistent with eq. (1.3) [17], and we can approximately confirm this behaviour, see Figure 2 . The exponent is not singled out very precisely, but the essential observation is the absence of a Banks-Casher type plateau in the total eigenvalue density near 0 .

Nevertheless, we observed an amazing connection to chiral RMT with respect to the ratio of $\left\langle\lambda_{1}\right\rangle$ in the sectors with topological charge $|v|=0$ and 1. We illustrated in Ref. [11] the chiral condensate $\Sigma$ as a function of this ratio at various masses, according to chiral Random Matrix Theory [16]. The combination of this RMT relation with $\Sigma(m)$ in eq. (1.3) enables us to eliminate the chiral condensate and to arrive at a prediction for the ratio

$$
\frac{\left\langle\lambda_{1,|v|=1}\right\rangle}{\left\langle\lambda_{1, v=0}\right\rangle}(m)
$$

which can be directly confronted with the numerical data in Table 1, see Figure 3. 

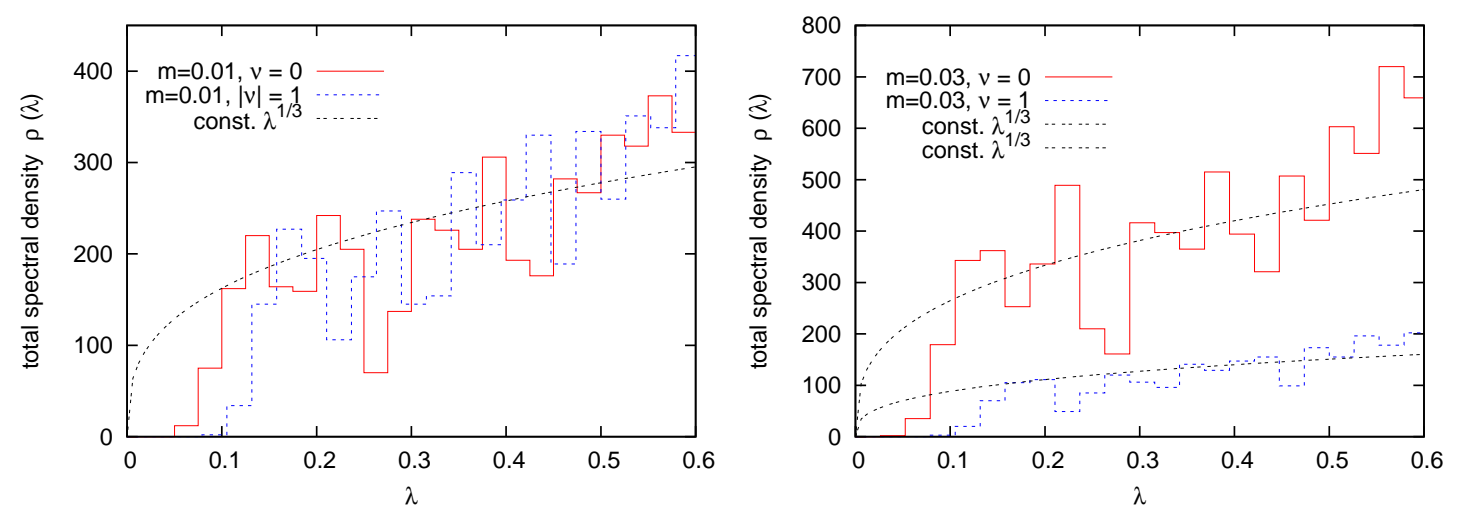

Figure 2: Histograms for the total eigenvalue density $\rho(\lambda)$ near zero for our two lightest fermion masses. The data are consistent with the expected behaviour $\rho(\lambda) \propto \lambda^{1 / 3}$, and we see a wiggle structure in addition.

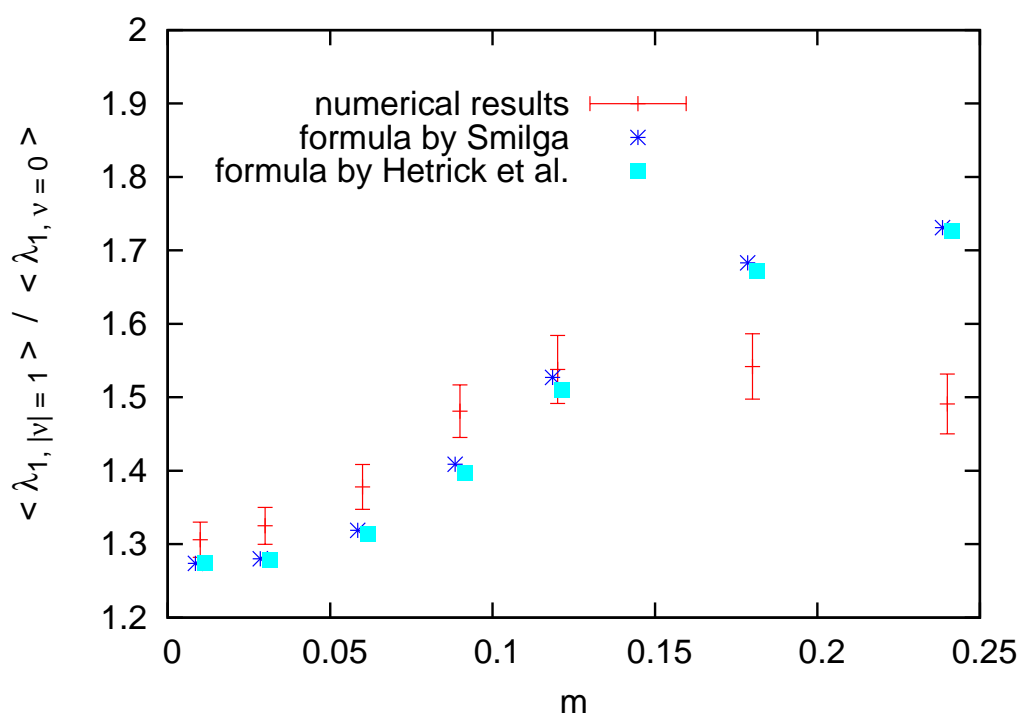

Figure 3: The results for the eigenvalue ratio of the leading non-zero Dirac eigenvalues in the topological sectors 0 and $1,\left\langle\lambda_{1,|v|=1}\right\rangle /\left\langle\lambda_{1, v=0}\right\rangle$. Our data are compared with the theoretical prediction based on a combination of chiral RMT in the $\varepsilon$-regime and analytical formulae for $\Sigma(m)$ from Refs. [2, 3].

The simulation results reveal a significant dynamical effect. For masses $m \gtrsim 0.15$ we take a step towards the $p$-regime behaviour (insensitivity to $v$ ) and the condition $m \ll \beta^{-1 / 2}$ is not on solid grounds anymore. But for $m \leq 0.12$ the data match the predictions remarkably well (at very light masses the measured ratio tends to be just slightly above the prediction), although the latter combines apparently incompatible ingredients from chiral RMT the $\varepsilon$-regime and from infinite volume. This result can be compared to a study using quenched re-weighted configurations with the standard overlap operator [18]: that study obtained $\Sigma(m \rightarrow 0) \rightarrow 0$ and a behaviour consistent with $\Sigma \propto m^{1 / 3}$ at large masses, but the proportionality constant was not reproduced and the proportionality could not be observed at small masses. In our case, Figure 3 is sensitive to both, the exponent and the proportionality constant in eq. (1.3), and both are confirmed well. 


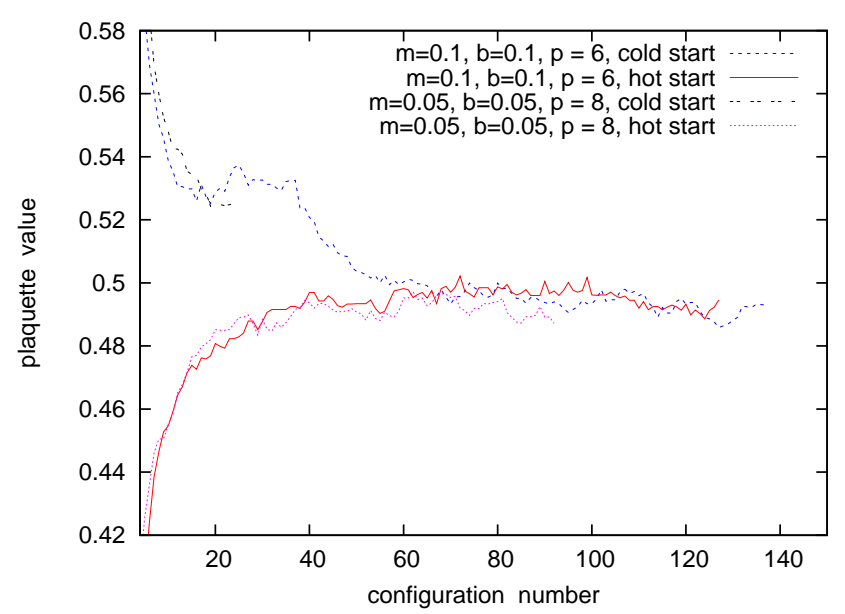

Figure 4: A thermalisation history for the dynamical overlap-HF in QCD at $\beta=5.6$ on a $8^{4}$ lattice. The algorithm is driven by the full HF force — corrected by a Zolotarev polynomial — and the trajectory length is 1 .

\section{Conclusions}

The overlap hypercube fermion has some computational overhead compared to the standard overlap fermion, but a number of benefits: better locality, approximate rotation symmetry, improved scaling and the applicability of a simplified HMC force. The restriction to low polynomials is particularly favourable for the numerical stability.

In our application to the 2 -flavour Schwinger model on a $16 \times 16$ lattice at $\beta=5$ we obtain useful acceptance rates and reliable reversibility. We cumulated statistics at masses $m=0.01,0.03$, $0.06,0.09,0.12,0.18$ and 0.24 in the sectors of topological charge $|v|=0$ and 1 . We revealed a new type of microscopic Dirac spectrum, which is not explored analytically. Nevertheless, by combining RMT formulae for the spectrum with analytical expressions for $\Sigma$, we obtained a prediction for the mass dependence of the ratio $\left\langle\lambda_{1,|v|=1}\right\rangle /\left\langle\lambda_{1, v=0}\right\rangle$, which matches our numerical data at $m \leq 0.12$ impressively well.

\section{A. Testing the dynamical overlap-HF in QCD}

We also implemented the HMC algorithm for $D_{\text {ovHF }}$ in QCD, with the HF force which can be chirally corrected with Zolotarev polynomials of any degree $p$. We display thermalisation histories of the dynamical overlap-HF in QCD, on $8^{4}$ lattices with polynomial degrees $p=6$ and 8 . They are applied to the HMC force in the spectral interval $\left[b, \lambda_{\max }\right]$ with a lower bound $b=0.1$ or $0.05\left(\lambda_{\max }\right.$ is the maximal Hermitian eigenvalue). For the precision parameter in eq. (3.2) we chose $\varepsilon=10^{-4}$, the trajectory length now amounts to $\tau=1$, and the accept/reject step is kept on machine precision.

At $\beta=5.6$ thermalisation sets in without problems (see Figure 4), whereas $\beta=5.7$ is plagued by a first order phase transition, which is further pronounced at $\beta=5.8$ (Figure 5).

Acknowledgement : We are indebted to Poul Damgaard, Stephan Dürr, Martin Hasenbusch, Urs Heller, Jacques Verbaarschot and Tilo Wettig for helpful discussions. J.V. was supported by the "Deutsche Forschungsgemeinschaft" (DFG). The computations were performed on the p690 clusters of the "Norddeutscher Verbund für Hoch- und Höchstleistungsrechnen" (HLRN). 

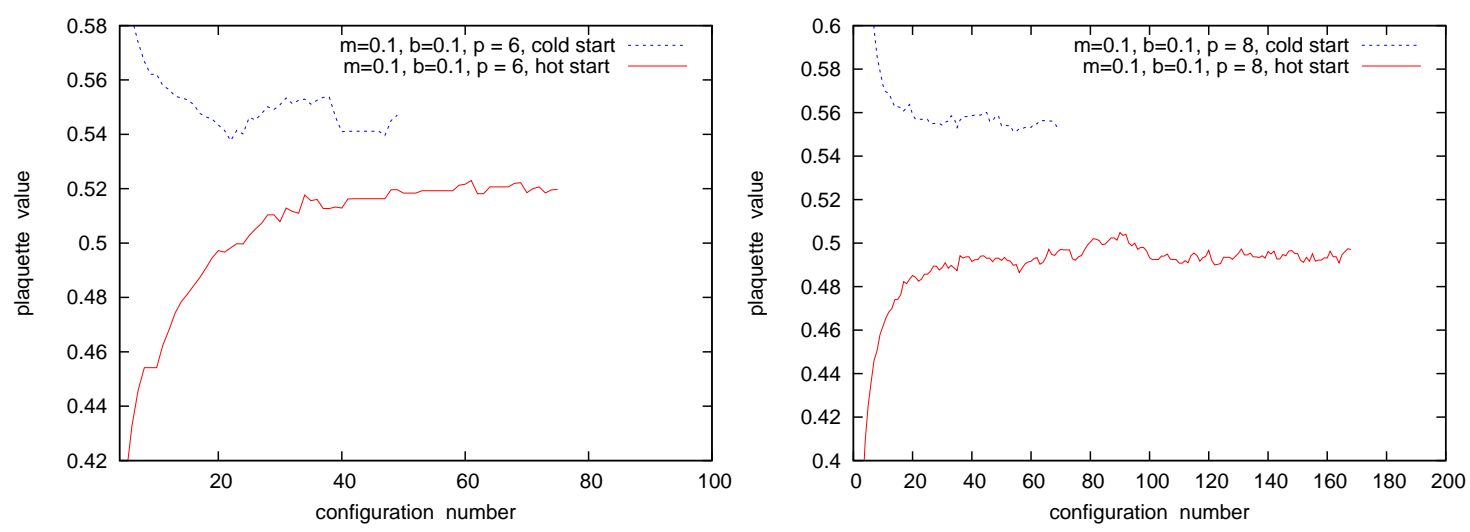

Figure 5: Thermalisation history for the dynamical overlap-HF in QCD on a $8^{4}$ lattice at $\beta=5.7$ (on the left) and $\beta=5.8$ (on the right). In contrast to $\beta=5.6$ (Figure 5) the trajectories for cold and hot starts level out on different plateaux, which indicates a fi rst order phase transition.

\section{References}

[1] J. Schwinger, Phys. Rev. 128 (1962) 2425. S.R. Coleman, R. Jackiw and L. Susskind, Annals Phys. 93 (1975) 267. S.R. Coleman, Annals Phys. 101 (1976) 239.

[2] J.E. Hetrick, Y. Hosotani and S. Iso, Phys. Lett. B350 (1995) 92.

[3] A.V. Smilga, Phys. Rev. D55 (1997) 443.

[4] H. Neuberger, Phys. Lett. B417 (1998) 141; Phys. Lett. B427 (1998) 353.

[5] W. Bietenholz, Eur. Phys. J. C6 (1999) 537.

[6] W. Bietenholz and I. Hip, Nucl. Phys. B570 (2000) 423.

[7] W. Bietenholz, Nucl. Phys. B644 (2002) 223. S. Shcheredin, Ph.D. Thesis, [hep-lat/0502001]. W. Bietenholz and S. Shcheredin, Nucl. Phys. B754 (2006) 17.

[8] S. Shcheredin and E. Laermann, PoS(LAT2006)146.

[9] N. Christian et al., Nucl. Phys. B739 (2006) 60.

[10] W. Bietenholz and H. Dilger, Nucl. Phys. B549 (1999) 335.

[11] J. Volkholz, W. Bietenholz and S. Shcheredin, PoS(LAT2006)040.

[12] J.C. Sexton and D.H. Weingarten, Nucl. Phys. B380 (1992) 363.

[13] N. Christian et al., PoS(LAT2005)239.

[14] W. Bietenholz, K. Jansen and S. Shcheredin, JHEP 0307 (2003) 033. L. Giusti et al., JHEP 11 (2003) 023. D. Galletly et al., Nucl. Phys. B (Proc. Suppl.) 129\&130 (2004) 456.

[15] T. DeGrand, Z. Liu and S. Schaefer, Phys. Rev. D74 (2006) 094504. H. Fukaya et al. (JLQCD Collaboration), Phys. Rev. Lett. 98 (2007) 172001; arXiv:0705.3322 [hep-lat].

[16] T. Wilke, T. Guhr and T. Wettig, Phys. Rev. D57 (1998) 6486. P.H. Damgaard and S.M. Nishigaki, Phys. Rev. D58 (1998) 087704; Phys. Rev. D63 (2001) 045012.

[17] P.H. Damgaard, U.M. Heller, R. Narayanan and B. Svetitsky, Phys. Rev. D71 (2005) 114503.

[18] S. Dürr and C. Hoelbling, Phys. Rev. D69 (2004) 034503. 